\title{
Tipología de la diplomacia pública de ciudades
}

\author{
Juan Luis Manfredi Sánchez \\ juan.manfredi@uclm.es
}

Universidad de Castilla-La Mancha.

Recibido: 13 de agosto de 2018 .

Aceptado: 23 de abril de 2019.

\section{Resumen}

La diplomacia pública de ciudades es la actividad de comunicación política internacional que más ha crecido en la última década, como consecuencia de la nueva estructura demográfica, la transformación del poder político y económico, el impacto del cambio climático y el empoderamiento ciudadano. Las ciudades, como actores privilegiados en la arena internacional, influyen en los asuntos globales, tales como las infraestructuras y tecnologías de la información, el turismo, la salud pública y la lucha contra el cambio climático. Las ciudades que aspiran a ser globales han entendido que su valor reside en la capacidad de apalancar poder y población, dar continuidad a las políticas públicas y crear capital simbólico a través de las experiencias directas y mediadas por los medios de comunicación y cultura. El presente estudio explica en qué consiste la particularidad de la diplomacia pública urbana y cuáles son sus distintas herramientas (marca territorio, creación de redes de influencia o protección de intangibles), y examina numerosas iniciativas de diplomacia pública en tres ejes: en primer lugar, la promoción de la economía e internacionalización de la industria; en segundo lugar, la representación política e influencia en asuntos globales; por último, la práctica de la lengua y la cultura, y su relación con la identidad. Se concluye que las ciudades, organizadas en red, han innovado en la práctica de la diplomacia pública, aunque no sean titulares efectivas de esta competencia internacional.

Palabras clave: diplomacia pública, ciudad, gestión, nuevos poderes.

\section{The typology of city public diplomacy}

\begin{abstract}
City public diplomacy has, in the past decade, become the fastest-growing activity in international political communication. This has been the result of new demographic structures, the transformation of political and economic power, the impact of climate change, and the empowerment of citizens. Cities, as privileged actors in the international arena, influence global affairs, including information infrastructure and technology, tourism, public health, and the fight against climate change. Cities with global aspirations have understood that their value resides in their capacity to leverage power and population, give continuity to public policies, and create symbolic capital via experiences that can be both direct and mediated by culture and communication media. The present study identifies the specific features of urban public diplomacy and its various tools (territory brands, the creation of webs of influence or the protection of intangibles), and examines numerous public diplomacy initiatives from three perspectives: economic deve-
\end{abstract}


lopment and the internationalization of industry; political representation and influence on global affairs; and language and culture, and their relationship to identity. We conclude that cities, organized as a web, have innovated in the field of public diplomacy, though they may not be at the forefront of this international competition

Keywords: public diplomacy, city, management, new powers.

\section{Tipologia da diplomacia pública das cidades}

\section{Resumo}

A diplomacia pública das cidades é a atividade de comunicação política internacional que mais cresceu na última década, como conseqüência da nova estrutura demográfica, da transformação do poder político e econômico, do impacto das mudanças climáticas e do empoderamento dos cidadãos. As cidades, como atores privilegiados na arena internacional, influenciam questões globais como infraestrutura, turismo, saúde pública e o combate às mudanças climáticas. As cidades que aspiram a ser globais entenderam que seu valor reside na capacidade de alavancar poder e população, dar continuidade a políticas públicas e criar capital simbólico por meio de experiências diretas e por aquelas facilitadas pela mídia e a cultura. O presente estudo explica qual é a particularidade da diplomacia pública urbana e suas diferentes ferramentas (território marca, criação de redes de influência ou proteção de intangíveis), examina numerosas iniciativas de diplomacia pública em três eixos: promoção da economia e internacionalização da indústria, representação política e influência em questões globais e a prática da linguagem e da cultura e sua relação com a identidade. Conclui-se que as cidades, organizadas em rede, tem inovado na prática da diplomacia pública, embora não sejam detentoras efetivas dessa competição internacional.

Palavras chave: diplomacia pública, cidade, gestão, novos poderes.

\section{Introducción y fundamento ${ }^{1}$}

La Nueva Agenda Urbana es resultado de la conferencia Hábitat III, celebrada en Quito en 2016. Recoge las prioridades de las políticas públicas de las ciudades, en colaboración con los Estados, los donantes, la sociedad civil y otros actores interesados. Plantea una agenda de trabajo sobre cinco pilares: 1) el fortalecimiento del papel de los gobiernos locales; 2) la apuesta por soluciones creativas y prácticas innovadoras; 3) la construcción de alianzas inclusivas y la participación ciudadana; 4) la adopción de un modelo de desarrollo territorial integrado y sostenible, y 5) el monitoreo, la promoción de la información pública y la evaluación del impacto de las políticas públicas. Con estos elementos, se establecen los retos que se enumeran a continuación:

La Nueva Agenda Urbana debe asegurar el desarrollo de capacidades de las autoridades locales y la descentralización del poder y los recursos hacia los gobiernos locales y las comunidades que lideran, a fin de que puedan convertirse en actores más protagónicos de la planificación, financiación e implementación de la política de desarrollo sostenible (Asamblea General de Socios [GAP, por su sigla en inglés], 2016, p. 13).

\footnotetext{
La investigación que dio lugar a este artículo cuenta con financiación del proyecto "La diplomacia pública de las megaciudades iberoamericanas: estrategias de comunicación y poder blando para influir en la legislación ambiental global" (RTI2018-096733-B-I00).
} 
La Agenda actualiza la conferencia Hábitat II, celebrada en 1996 y orientada a la sostenibilidad de los asentamientos humanos en sintonía con los Objetivos de Desarrollo del Milenio de Naciones Unidas. Se sostiene sobre cinco tesis de gobernanza que explican cómo ha cambiado el planeta desde 1996. En primer lugar, se afirma que la urbanización es un proceso imparable. Alrededor del $60 \%$ de la población ya vive en núcleos urbanos y el $25 \%$, en megaciudades o corredores que aglomeran una cadena de núcleos, como el eje Manchester-Milán, el Gran Estambul o el Delta del Río Perlas en China. La acumulación demográfica está localizada y responde a la concatenación de factores económicos e infraestructuras. Al mismo tiempo, genera nuevas desigualdades de acceso, empleo y riqueza. Se estima que en torno al $80 \%$ de las nuevas aglomeraciones se darán en África y Asia (United Nations, Department of Economic and Social Affairs, Population Division, 2018).

La segunda tesis de gobernanza es la transformación del poder (Mendoza y Vernis, 2008). Se ha diluido la capacidad para imponer decisiones de forma unilateral en un mundo cada vez más inestable, al tiempo que han aparecido nuevas formas de organización política que amenazan el Estado nación convencional. La política, las instituciones y el contrapoder se han refugiado en las ciudades, entorno que reclama para sí más participación en la globalización.

Ramió (2016) considera que la crisis del Estado ha afectado la conceptualización misma del ejercicio del poder y que las estructuras de poder y administración agrupan las relaciones entre el poder político, los gobiernos, el mercado y la sociedad civil en nuevos repartos geográficos, tales como el local, el regional, el macrorregional o el multilateral. Este divorcio (Bauman y Bordoni, 2016) entre política real y poder es esencial para comprender por qué las ciudades aspiran a dominar su propia agenda de temas.

En el plano político, interesa la irrupción de start-ups políticas que responden a la desafección de la política convencional, apuestan por liderazgos fuertes sin estructuras de partido y manejan las redes sociales para colocar sus mensajes (Manfredi Sánchez, 2018). Por su sincretismo ideológico, son el espacio natural para el hiperliderazgo, puesto que ofrecen soluciones generalistas y compromisos flexibles para un "votante flotante" (Manin, 1998, p. 285). Sin estructura de partido, se refuerza el carisma individual del alcaldable, que busca proyección internacional.

El tercer eje es la transformación económica. En el ámbito público, la deuda y la crisis fiscal son una constante desde los 70. Los Estados no son capaces de atender todas las demandas de la macropolítica y han delegado en instituciones políticas no estatales la acción pública en numerosas materias, tales como sanidad, educación, transporte, talento y universidades. Asimismo, las nuevas geografías digitales han creado un universo conectado a través de los bytes y los dispositivos. La digitalización impacta en las cadenas de suministros, que se soportan sobre infraestructuras conectadas a través 
Juan Luis Manfredi Sánchez

Tipología de la diplomacia pública de ciudades

del comercio global. En el ámbito de los servicios, la irrupción de nuevos operadores ha afectado al turismo, al transporte de viajeros, al sector retailer o al consumo de ocio. La economía de la innovación se organiza en hubs y clusters, que conectan ciudades, antes que territorios nacionales, de modo que es posible identificar ciudades globales (Sassen, 1991), que articulan la producción económica y los mercados financieros. En el ámbito industrial, las urbes concentran las compañías, los reguladores y los grupos de presión que se dedican a actividades interrelacionadas. Actúan como anclas de una industria tecnológica de alto nivel que necesita organizarse en mercados regionales para competir en la globalización. Por este motivo, se convierten en polos de referencia para crear entornos competitivos. Londres, Estocolmo, Boston y Silicon Valley compiten por el liderazgo en el ámbito de la economía digital. Ofrecen diferentes propuestas de valor, que tienen en común el deseo de liderar la nueva generación de desarrollos tecnológicos. En este plano, se observan políticas para la atracción del talento tecnológico y la gestión de la reputación, para ser percibidas como ciudades dinámicas, bien conectadas y abiertas al cambio, como explica Florida (2003). La clase creativa, en palabras de este sociólogo, lidera la transformación urbana, la creación de un nuevo modelo económico basado en la innovación, y ha sabido aprovechar las ventajas de la globalización. La ciudad responde mejor y de forma más ágil a las necesidades de cambio permanente.

La cuarta dimensión consiste en el cambio climático, que modifica las reglas de juego. La influencia humana en el planeta tiene características irreversibles, que afectan al ecosistema. Se trata de un debate enorme que empieza en las ciencias duras y acaba en el diseño (como el Madrid Design Festival), las ciencias sociales (Arias Maldonado, 2016) y la ficción (como las novelas de Ballard o Cormick). En el plano urbano, nos interesa porque el impacto humano en el clima altera la distribución de la población, las fuentes de riqueza y materias primas, la gestión del poder y las políticas urbanísticas (Acuto, 2016). Este enfoque de respeto por el planeta aparece en Hábitat III como un elemento definidor de principios y valores:

La Nueva Agenda Urbana debe aceptar que la ciudad y el proceso de gestión y desarrollo urbanos existen y tienen lugar en un marco de desarrollo sostenible que los reconoce como un subconjunto de la sociedad, la economía y el medio ambiente (Acuto, 2016, p. 612 , traducción propia).

El último elemento de la serie es el más significativo para nuestro estudio sobre la diplomacia pública de las ciudades. Los ciudadanos, organizados en torno a movimientos sociales, han liderado el debate sobre las identidades y la agenda política alejados de las instituciones (sindicatos, partidos) y las estructuras convencionales (Estado nación). En la ciudad, encuentran acomodo estas reivindicaciones de geografía política (Harvey, 2013). El uso de medios y redes sociales ha afectado la forma 
de organización, los instrumentos de comunicación y participación, la capacidad de impacto en grandes audiencias y la validación de las demandas. Los movimientos sociales de naturaleza política no pueden competir en estas dimensiones mediáticas con las estructuras consolidadas de los Estados. Esta debilidad estructural ha dado lugar al activismo mediático que organiza actividades de bajo presupuesto en las ciudades -a veces, simultáneamente en varias ciudades del mundo- que persiguen la creación de nuevas prácticas comunicativas, con el fin de influir en el discurso dominante o disminuir el poder de los medios convencionales mediante el establecimiento de una agenda política y mediática contrahegemónica (Carroll y Hackett, 2006). Las actividades digitales han incrementado las capacidades de acción colectiva en la medida en que "han afectado a la agenda pública, al activismo y a la participación política en la arena internacional" (Manfredi Sánchez, 2014, p. 343). Para Melucci (1989), la creación de símbolos y relaciones sociales ha reemplazado la producción material de la protesta, por lo que se han eliminado las fronteras entre el activismo y el activismo de naturaleza mediática.

En la agenda de preocupaciones, encontramos el cambio climático, el transporte, la vivienda, la salud pública global, la alimentación y otros asuntos que escapan a la práctica convencional de la política exterior de los Estados. Por su naturaleza, estos problemas no entienden de fronteras geográficas. El empoderamiento ciudadano identifica tres usos de la comunicación pública en la ciudad (Lemmert, 1981): la movilización, la apertura de nuevos espacios de comunicación y la creación de fuentes informativas alternativas mediante campañas de divulgación, sensibilización y promoción de nuevos comportamientos sociales.

En este contexto, nos planteamos las siguientes preguntas de investigación: ¿cómo han desarrollado las ciudades sus estrategias de diplomacia pública en el marco de una gobernanza multinivel? ¿Alinean las ciudades sus estrategias con los gobiernos estatales o se trata de iniciativas independientes? ¿Constituyen las ciudades redes y alianzas como instrumentos para la promoción de sus intereses?

El objetivo general de la investigación es conocer los recursos, los rasgos y las prácticas de la diplomacia pública que realizan las ciudades, de modo que se conozcan los fines, los mecanismos de colaboración público-privada y el grado de incidencia política en asuntos internacionales. El trabajo da lugar a numerosas preguntas: ¿hasta qué punto las estrategias se alinean con el interés de los Estados? ¿Cómo se organiza el activismo para mejorar su capacidad de incidencia política? ¿De qué modo la gobernanza del cambio climático empodera a la ciudad en la gobernanza multinivel? Nuestra contribución se estructura en torno a tres hipótesis:

1. Las ciudades son actores principales en el desarrollo de la diplomacia pública como campo de la comunicación política internacional. Su actividad encaja con 
las definiciones clásicas (Cull, 2008; Nye, 2003; Seib, 2012), aunque no sean titulares de la competencia en materia de política exterior.

2. La diplomacia pública de las ciudades se ha multiplicado porque cuenta con una agenda más amplia de intereses y porque la falta de competencias reales (duras) le confiere más flexibilidad en el diseño y la ejecución de campañas de comunicación. El valor de sus acciones encaja con la gestión de intangibles y la reputación.

3. El gobierno local ofrece soluciones concretas, reales y efectivas, cercanas a la experiencia del ciudadano. No se trata de experiencias mediadas, sino protagonizadas por él. La incidencia de la política municipal en la gestión y la regulación multinivel está en el corazón de la agenda de investigación (Barber, 2013; Curtis, 2014; Schragger, 2016). Por eso, se observa una geometría variable de relaciones en red, no jerarquizadas.

\section{Definición y gestión estratégica de la diplomacia pública de ciudades}

\section{Diplomacia pública e influencia global}

La diplomacia pública consiste en la gestión de la comunicación política internacional con arreglo a unos intereses de acción y política exterior. Tiene como objetivo "el ejercicio de la influencia por parte de una entidad sobre un público extranjero mediante el desarrollo de estrategias de comunicación, que incluyen los programas educativos, informativos y de entretenimiento" (Manfredi Sánchez, 2011, p. 211). La influencia es un concepto ambiguo, polisémico, que se define por un acuerdo de mínimos. Se trata de la capacidad de modificar el curso de una acción, sea por mediación del gobierno, la opinión pública o las iniciativas privadas. Para la diplomacia pública, se concreta en el cambio en las percepciones que se tienen de un territorio, el incremento de la demanda de bienes y servicios exportados, el deseo de aprender un idioma o cualquier otra dimensión económica, política o social que se mida en una cuenta de resultados.

La literatura académica ha diferenciado entre la diplomacia convencional, que explica la conducción de las relaciones por parte de un Estado dentro del sistema internacional, y la pública, aquella que de manera expresa promueve el intercambio de ideas y capital simbólico, productos y servicios culturales, el diálogo directo entre ciudadanos y la elusión de intermediarios (Gonesh y Melissen, 2005; Seib, 2012). La diplomacia pública tiene como meta el establecimiento de un marco de comprensión y de decisión en un entorno cambiante, y la configuración de una ventaja a través de la comunicación (Cull, 2008). Los cinco componentes de la estrategia, según el profesor Cull (2008), 
son los siguientes: la capacidad de escucha, la promoción de intereses, la diplomacia cultural, los intercambios educativos y las emisiones internacionales de radio y televisión. Por este motivo, la diplomacia pública se ha convertido en el paradigma de referencia para comprender la comunicación política: "Los estudios sobre diplomacia pública construyen un puente entre los estudios sobre la política y la comunicación internacional con la teoría de las relaciones internacionales" (Ociepka, 2018, p. 290).

La diplomacia pública es transparente en su diseño y ejecución, persigue el entendimiento mutuo, conecta con un objetivo de gobierno y su vía de financiación es conocida. Aspira a la creación de confianza entre las partes y la rendición de cuentas al público propio y extranjero, si bien "el crecimiento de la transparencia no ha disminuido el efecto de la propaganda en sociedades con menores índices de confianza social" (Manfredi Sánchez, Herranz de la Casa y Calvo Rubio, 2017, p. 837). En el plano procedimental, se emplea para explicar los procesos, las negociaciones o los acuerdos. La transparencia ha facilitado el acceso de nuevos actores e interesados en la definición de las políticas internacionales, en la medida en que se conocen las agendas, los propósitos y las campañas.

La diplomacia pública es una política pública, y no una mera campaña de mercadeo o reposicionamiento publicitario. No debe confundirse con el concepto de propaganda, cuya finalidad es influir en la opinión pública extranjera sin reconocimiento expreso del gobierno. El uso propagandístico no ha disminuido con la emergencia de la diplomacia pública, sino que se ha sofisticado: "El espacio global de la información se caracteriza por plataformas peer-to-peer y conversación, debate y diálogo antes que por exhortaciones unidireccionales y mensajes resonantes de comunicación en tiempos de guerra o propaganda" (Robinson, Helmus, Cohen, Nader, Radin, Magnuson y Migacheva, 2018, p. xx, traducción propia).

La diplomacia pública encaja en el desarrollo de la actividad internacional, que requiere una interpretación abierta de la realidad. El poder se ha diluido en la medida en que hay más actores con capacidad de ejecución global, más intereses cruzados y más redes de colaboración y, a la vez, competencia. En particular, la comunicación política internacional se ha multiplicado por la liberalización de los mercados informativos, la expansión de las radios y las televisiones globales, la irrupción de Internet, la emergencia de las redes sociales y el empoderamiento ciudadano a través de las tecnologías. En este contexto, la diplomacia pública aparece como una oportunidad para el ejercicio del poder en la arena de la comunicación con el ánimo de influir en la percepción de la opinión pública, la configuración de los hechos noticiosos y la capacidad de influencia.

La influencia requiere de la colaboración y la cocreación de gobierno. En la materia que nos interesa, es un proceso abierto en el que confluyen todos los intereses geográficos (regiones, ciudades) y sectoriales (ONG y tercer sector, empresas familiares 
Juan Luis Manfredi Sánchez

Tipología de la diplomacia pública de ciudades

y pymes, universidades y medios de comunicación, productores de materias primas y consumidores). La actividad digital de los nuevos influyentes (Del Fresno, Daly y Segado, 2016), la diplomacia privada y corporativa, el lobby, la relación con los think tanks, el empoderamiento ciudadano, la diplomacia parlamentaria o la propia internacionalización de la economía y la empresa constituyen oportunidades para impulsar la proyección exterior de la ciudad, no solo mediante el ejercicio diplomático convencional a través del Estado.

En el ámbito diplomático, existe un rico debate sobre los actores y sus capacidades (Cornago, 2013). Cualquier actor con capacidad de obrar en la esfera internacional participa de hecho dentro y fuera de los círculos convencionales de acción diplomática, política y comunicativa. Y la lista se extiende cada día: empresas tecnológicas, multinacionales, fondos de inversión, ciudadanos a título individual, instituciones y organismos internacionales, regiones o ciudades reclaman su espacio en la arena internacional. En un trabajo pionero, Van der Pluijm (2007) estipula que la diplomacia de ciudades es el conjunto de instituciones, procedimientos, acciones e iniciativas urbanas que permite a las ciudades relacionarse con otros actores en el escenario internacional; mediante esta acción se representan a sí mismas, sus intereses y el de sus ciudadanos. Como señala Copeland (2009), los nuevos actores emplean técnicas de guerrilla para colocar sus mensajes y vehicular sus preferencias ante la opinión pública global.

La diplomacia pública es la expresión de la pérdida de monopolio en el ejercicio de la comunicación internacional, independientemente de la titularidad del derecho internacional. Setzer explica que las nuevas áreas de la gobernanza global no encuentran cabida en el ordenamiento jurídico nacional por sí solo:

\begin{abstract}
De forma habitual, no hay leyes nacionales, o al menos tienen una base doméstica legal débil, en relación con la diplomacia subestatal. A pesar de esta relativa debilidad legal, los principios de iure pueden ser sustituidos por prácticas de facto. Un asunto clave es determinar si la acción subestatal cae en el área tradicional de la competencia estatal. Si las acciones subestatales violan las responsabilidades de gobierno estatal en materia de seguridad nacional, defensa, asuntos internacionales o préstamos internacionales, su implicación en relaciones internacionales puede ser denunciada ante la justicia (2015, p. 333 , traducción propia).
\end{abstract}

No desaparece el Estado nación, pero sí se observan nuevos yacimientos de poder que emplean la comunicación internacional para reputarse, influir o crear la ilusión de participar. Este planteamiento estratégico de gobierno, relacionado con el principio de subsidiariedad, está en el espíritu de Hábitat III y perfila el rol de las autoridades locales, que "asumen la gran responsabilidad de suministrar servicios e implementar políticas fundamentales de desarrollo urbano sostenible, sobre lo cual deben rendir cuentas" (GAP, 2016, p. 13). 
Austral Comunicación

Volumen 8, número 1 (junio de 2019): 37-62. ISSN 2313-9129

\section{El valor de la ciudad}

Entre tanta complejidad, solo una institución crece: la ciudad. La multiplicidad de fuentes de legitimación, participación y comunicación confirman la teoría de una diplomacia pública y la oportunidad de la ciudad en el nuevo escenario. La diplomacia urbana ha multiplicado de forma exponencial las cinco funciones clásicas atribuidas a la dirección de asuntos internacionales: la comunicación, la negociación de acuerdos, la gestión de la información, la prevención de conflictos y la expresión simbólica de una sociedad internacional (Bull, 1995).

Las ciudades conforman el corazón de la nueva diplomacia pública porque presentan tres cualidades exclusivas y diferenciadoras en la globalización: apalancamiento, continuidad y capital simbólico. Son organizaciones flexibles, cuya elasticidad permite el empleo de instrumentos no convencionales y la interconexión con otros participantes de la denominada diplomacia en red (Cooper, Heine y Thakur, 2013). La ciudad se identifica como nodo de la sociedad red (Castells, 2009), que sobrevive gracias a las características de flexibilidad, escalabilidad y supervivencia, los tres principios que plantea Castells (2009).

En relación con el apalancamiento, la capacidad para agregar y aunar población, gobierno, iniciativa privada, talento, creatividad, infraestructuras y transportes es propia de las urbes. Se ha globalizado la biografía, pero no la residencia. Nuestro hogar se ubica en una ciudad concreta, un barrio insertado en la gran metrópoli. En esta se aglomeran las actividades económicas e industriales basadas en la innovación, porque solo allí se pueden concentrar las inversiones, los desarrollos económicos, la demanda de consumo, las universidades y los centros de investigación, así como el capital humano, el talento. El apalancamiento se beneficia de los efectos de la economía de redes. Las ciudades se conectan a través de aeropuertos e infraestructuras tecnológicas, que eliminan las barreras del Estado y facilitan la colaboración en el desarrollo de nuevos proyectos. En el ámbito económico, se manifiesta en la globalización intensiva, concentrada en los núcleos urbanos, y no en las poblaciones rurales. Las urbes condensan la población y la actividad humana, de modo que conforman el tejido de producción, distribución y consumo.

El segundo elemento es la continuidad. Consiste en la identificación de espacios urbanos con centenares, quizás milenios, de actividad y ocupación a lo largo de todo el planeta. Estambul, Moscú, Roma, Jerusalén o Londres ocupan el mismo espacio y pueden reivindicar un estatus propio. Son ciudades que están asentadas en la Historia, de modo que representan una línea de continuidad, una secuencia lógica de desarrollo territorial, de orden geográfico. La característica en cuestión facilita la creación de redes y rutinas, y la posibilidad de especialización. La ciudad crece o decrece, según los ritmos económicos, pero por su dimensión es una organización escalable. La escalabi- 
lidad se manifiesta en la capacidad de crecer de forma exponencial sin incrementar los costes de producción, esto es, los recursos destinados a la consecución de los objetivos de la política exterior. Por eso, la diplomacia de ciudades se organiza en redes que facilitan la cooperación de terceros. Para Zeraoui y Castillo Villar (2016), estas estrategias son propias de la paradiplomacia, que articula redes de cooperación y colaboración de naturaleza urbana. Como explica Setzer:

\begin{abstract}
Las redes de ciudades, como la red C40, aspiran a impactar directamente en otras esferas de gobernanza global mediante la influencia en las dinámicas públicas internacionales y nacionales (e.g. organizando encuentros internacionales, haciendo lobby en el gobierno central, o participando en los foros internacionales). En este nuevo escenario diplomático, los gobiernos locales y regionales han empezado a llamar a la introducción de una nueva categoría de "grupo de interesados gubernamentales" entre los observadores acreditados ante el sistema de Naciones Unidas (2015, p. 331, traducción propia).
\end{abstract}

A su vez, según apuntan Acuto, Morissette y Tsouros:

La internacionalización de las redes de ciudades no está siendo impulsada solamente por
las organizaciones multilaterales, sino también por influyentes miembros del sector priva-
do. Por encima del $63 \%$ de las redes "internacionales" de ciudades encuestadas por el Lab
tienen acuerdos multilaterales y corporativos con organizaciones como UNICEF, ILO,
Hábitat, o intereses privados como Google, SAP y Cisco, o con grupos filantrópicos como
Bloomberg Philanthropies y Rockefeller Foundation. Esta actividad es en particular rele-
vante en las redes a favor de la sostenibilidad, pero el escenario incluye también redes re-
lacionadas con la salud, la seguridad o bien otros sectores (2017, p. 16, traducción propia).

La continuidad es un activo en comunicación cuando se pueden reforzar intangibles vinculados con la memoria colectiva y el capital simbólico, el tercer valor de la ciudad. Este último consiste en la creación de narraciones, metáforas e historias que pasan al imaginario colectivo (Silva, 2006). Se concreta en el imaginario del cine, el arte y el patrimonio, las escenas culturales, la globalización de los patrones de ocio y consumo, y las representaciones estéticas. Por ejemplo, conocemos Manhattan antes incluso de aterrizar en Nueva York. Río de Janeiro quiere que Woody Allen cree y ruede una historia cinematográfica. Medellín quiere dejar de ser la ciudad de Pablo Escobar y, por eso, ha trabajado por ser la ciudad más sostenible e innovadora de América Latina, y se disgusta por el éxito de la teleserie Narcos en Netflix. También se encuentra capital simbólico en la arquitectura del poder y las relaciones internacionales. La experiencia de la ciudad es única y se sustenta sobre la libre elección de cada visitante, que organiza su viaje de acuerdo con sus prioridades. El capital simbólico incrementa el modelo asociativo de Zenker y Braun:

En la mente de los consumidores se crean unas asociaciones de ideas basadas en la expresión visual, verbal y en la experiencia, a través de las cuales se obtienen los objetivos, la comunicación, los valores y, en general, la cultura de los grupos de interés que diseñan o que afectan al diseño de un lugar, de un destino (2010, p. 33, traducción propia). 
La ciudad no es un circuito cerrado, sino una experiencia polisémica, relacionada con funciones sociales (Caldwell y Freire, 2004), un territorio y una identidad construida sobre sus elementos funcionales (infraestructuras, servicios básicos, transporte), experienciales (contacto con la gente, gastronomía, teatro, actividades en directo) y simbólicos (calidad humana, hospitalidad). En ese contexto de análisis, la experiencia de cada una contiene imágenes, fotografías, contacto con la población local, gastronomía y deporte, una suerte de supermarca construida sobre "una imagen geográfica no delimitada" (Choi y Cai, 2016). En su dimensión territorial, la ciudad se corresponde con una actividad de diplomacia pública subestatal que contribuye a la marca país (Wang, 2006).

En materia internacional, el capital simbólico es relevante para los gobiernos subestatales porque tiene capacidad de impacto en la opinión pública cercana (Meerts, 2015). Las ciudades contribuyen a la discusión de los asuntos internacionales en clave doméstica mediante conferencias, declaraciones, manifiestos, impulso de instrumentos no normativos y promoción de la marca territorio (Wang, 2006). En ese sentido, Hocking señala:

\begin{abstract}
la diplomacia se ha convertido en una actividad que consiste en la creación de redes, que abraza una amplia gama de estados y actores no estatales que se centran en la gestión de asuntos que demandan recursos sobre los cuales ningún participante por sí solo posee el monopolio (2006, p. 14, traducción propia).
\end{abstract}

La supervivencia pasa por la habilidad para reconfigurarse, buscar aliados y encontrar puntos de encuentro.

\title{
Taxonomía de la diplomacia pública de ciudades
}

Las ciudades ejercen la actividad política comunicativa internacional en tres ejes, a saber, la economía, la cultura y la política. Esta clasificación permite enumerar, catalogar y establecer patrones de comportamiento entre las acciones de diplomacia pública urbana. Como método de trabajo, definimos cada uno en un apartado y mostramos los ejemplos más pertinentes para la construcción de la teoría comunicativa de la diplomacia urbana. Si bien los casos seleccionados pueden evidenciar dos o más ejes, hemos decidido utilizarlos solamente en un apartado.

\section{Eje económico}

El eje económico responde a la necesidad de integrar la ciudad en el proceso de globalización como institución abierta a capitales y servicios internacionales. Aquí se incluyen las actividades conducentes a la promoción del comercio exterior, la internacionalización de la economía, la proyección de la empresa local y la gestión de la marca territorio. 
Juan Luis Manfredi Sánchez

Tipología de la diplomacia pública de ciudades

La gestión de la marca territorio y la marca ciudad tiene como finalidad la sobreexposición de unos atributos para una audiencia concreta: se busca captar inversiones, organizar eventos, incrementar el número de turistas, renovar la imagen, entre otros. La ciudad crea un universo simbólico dotado de significados. Las experiencias de marca territorio son variadas y orientadas a la creación de valor en el terreno de marketing estratégico, con la finalidad de proyectar una imagen exterior. Constituyen herramientas clásicas el logo, el diseño institucional y la señalética de la ciudad. Sobre estos cimientos, se crea una imagen permanente. Se trata de una técnica que genera mayores ventajas competitivas para la ciudad que para el Estado nación, ya que los monumentos (el Coliseo de Roma), las tradiciones culturales (la Semana Santa de Sevilla), los objetos (la cabina telefónica de Londres), la gastronomía (el queso Stilton) o los intangibles, como la música o la oralidad (la plaza central de Marraquech) se asocian a territorios concretos y definidos, no a un Estado de forma genérica. En este sentido, la ciudad puede optar por nuevas técnicas, tales como una campaña centrada en un activo o atributo concreto (visita al Museo Guggenheim de Bilbao, la última exposición de Picasso en París o el mayor centro comercial del mundo en Dubai; un megaconcierto en Woodstock; la recuperación de patrimonio en Palmira o en la ciudad monumental de Hatra; los festivales de teatro de Edimburgo o Mérida) o bien en una experiencia de destino, en la que el viajero organiza su propio trayecto e individualiza la experiencia: cada visitante decide cuánto caminar (Camino de Santiago), dónde parar o qué visitar.

En el mismo ámbito cabe encuadrar el turismo de congresos, cuyas cifras de visitantes y el impacto económico en la ciudad son esenciales en la construcción de la marca territorio. Según la International Congress and Convention Association (2018), Barcelona, que se ha especializado en la captación de eventos relacionados con la tecnología de la salud, lidera el número de congresos internacionales con 195 eventos, seguida por París, Viena, Berlín, Londres, Singapur y Madrid.

En materia de apertura e internacionalización, encontramos varios modelos de diplomacia urbana. En tecnología, destaca la competencia por la organización de eventos y foros de alcance mundial. Por ejemplo, el Barcelona Mobile World Congress reúne a más de 100000 profesionales del mundo tecnológico y es considerado un evento de primer nivel mundial, con un impacto económico de alrededor de 470 millones de euros en una semana. Las empresas digitales muestran sus avances, se prueban nuevas aplicaciones y se proyecta una imagen moderna de la ciudad, como destino referente para el turismo de encuentros académicos, la llegada de nuevas ferias, la ubicación de la ciudad en la agenda tecnológica. ${ }^{2}$

La actualidad política de Cataluña ha generado inquietud en John Hoffmann, el consejero delegado de la organización del Barcelona Mobile World Congress, quien plantea marcharse de Barcelona antes de que finalice el contrato en 2023 si persiste el riesgo político. Otras ciudades se han interesado por esta posibilidad y han procurado captar el interés del organizador. 
Austral Comunicación

Volumen 8, número 1 (junio de 2019): 37-62. ISSN 2313-9129

En el sector de servicios educativos, se han identificado hubs de educación superior, universidades y escuelas de negocios que aúnan la inversión en investigación y ciencia, el empleo de calidad, la mentalidad emprendedora y la apertura exterior. Su estructura agrega valor y no se limita a un municipio concreto, sino a corredores que ofertan educación, empresa y acceso al mercado global. Hay ciudades bien conectadas y con una larga trayectoria en la educación ejecutiva que también son sede preferente de un buen número de multinacionales. El ranking MBA City Monitor evalúa la capacidad de las ciudades y regiones para atraer talento global interesado en cursar algún programa de educación ejecutiva. Según él, en 2017, los diez primeros destinos son Boston, Nueva York, Londres, París, Chicago, Toronto, Barcelona, Carolina del Norte, Singapur y Filadelfia. Para Borafull, la lista de ciudades que atraen talento constituye un predictor útil para la gestión de los intereses internacionales de aquellas:

Las ciudades pueden utilizar para medirse y compararse adecuadamente en su capacidad de atraer y retener el talento global. Es importante resaltar por qué este puede ser un buen predictor [...] cursar uno de los programas MBA de las principales escuelas de negocios en el mundo supone un gran compromiso económico y humano para cualquier estudiante (2018, p. 46).

A mitad de camino entre la tecnología y la educación aparece la transformación digital; en ese sentido, la orientación emprendedora de las ciudades es un activo de comunicación internacional. Se persigue el establecimiento de nuevos negocios de base tecnológica, ya que se considera que estos son creadores netos de empleo, vehículos de modernización económica y generadores de transferencia tecnológica. Así, conforman ecosistemas emprendedores aquellos que consiguen reunir capital humano, acceso a los mercados y financiación. Otros indicadores de apertura a los flujos de economía internacional son la cooperación pública y privada en el plan Investigación+Desarrollo+Innovación $(\mathrm{I}+\mathrm{D}+\mathrm{I})$, la atracción de talento a través de una red de universidades, escuelas de negocios y espacios abiertos para el emprendimiento, la especialización para que el tejido empresarial tenga capacidad para diferenciarse, así como las facilidades para el capital semilla. Las ciudades compiten por posicionarse en ese mapa global, encabezado por el mito de Silicon Valley, a la que le han surgido competidores globales: Toronto, Montreal, San Pablo, Londres, Estocolmo, Berlín y Singapur han creado campañas de diplomacia pública para afectar la decisión de emprendedores que quieran instalarse en el país. Siguen la senda de Israel y su estrategia como start-up nation, que ha conseguido influir en el tejido productivo e industrial.

Por su parte, la economía de la cultura tiene peso específico en la diplomacia urbana. La cultura crea valor económico añadido porque alrededor de ella emergen las industrias editoriales, audiovisuales y musicales. En su desarrollo digital, encuentra acomodo en redes sociales, vídeos de YouTube o nuevos influyentes. Los eventos cul- 
turales, tales como los conciertos (el de Año Nuevo en Viena, con 1000 millones de espectadores audiovisuales en 70 países, por ejemplo) y los festivales (Sundance, San Sebastián o Cannes), generan expectación mediática y proyección internacional. La cultura sirve para transformar la imagen exterior y para ocupar nuevos espacios de comunicación, así como para crear nuevas industrias de actividad económica. Por ejemplo, el Festival Sónar aporta 124 millones de euros al PBI de la ciudad de Barcelona, divididos entre el impacto económico directo (más de 100000 visitantes procedentes de alrededor de 100 países, venta de entradas, instalaciones, conferencias, congreso Sónar $+\mathrm{D}$ de cultura digital) y el indirecto (formado por el sector de los servicios).

Las ciudades compiten por el establecimiento de sedes, entregas de premios y otras instituciones culturales que atraen rendimientos económicos directos. Lien y Lo evalúan los efectos positivos de los institutos culturales para la promoción de la lengua como vehículo de internacionalización de la economía, ya que traen consigo nuevas oportunidades de negocio y servicios de valor añadido al propio centro o instituto cultural:
Es importante señalar que un instituto cultural no se construye con el propósito de in- crementar el comercio y la inversión. Más bien, el principal propósito de cualquier insti- tución cultural es la mejora de la familiaridad con un idioma y el reconocimiento de una cultura en un intento de construir beneficios internacionales mutuos y relaciones en el ámbito de la cultura y la educación, así como la promoción de la amistad. Es razonable asumir que las personas que participan en una exhibición artística o una ópera organi- zada por un instituto cultural están interesadas en las áreas y la cultura, no en el negocio. Sin embargo, la información y las oportunidades de negocio están presentes a lo largo de dichas actividades y esto conduce a la mejora de los efectos económicos, como el incre- mento del comercio y la inversión extranjera (2017, p. 17, traducción propia).

Otro instrumento de diplomacia pública a mitad de camino entre la economía y la cultura es la oficina de captación de rodajes audiovisuales, denominada Film Commission. Su misión consiste en facilitar el trabajo a las productoras audiovisuales para que se instalen en una localidad y graben escenas, contenidos o exteriores. Con esta estrategia se dan a conocer a la audiencia global nuevos territorios, destinos o rutas culturales creadas ad hoc. Un ejemplo de esta práctica sucede en Andalucía. Las ciudades de Sevilla, Santiponce, Córdoba, Almodóvar del Río y Cádiz se han unido entre ellas para crear un servicio de información para el turista que quiere conocer y recorrer los escenarios y adentrarse en los rodajes o la intrahistoria de los numerosos proyectos ejecutados en la región. Según datos facilitados por la propia Andalucía Film Commission, los escenarios publicitados por la serie Juego de Tronos habían incrementado el número de visitantes en un $40 \%$.

Una mención especial merece la salida del Reino Unido de las instituciones europeas, porque ha generado externalidades en la diplomacia urbana. La salida de or- 
Austral Comunicación

Volumen 8, número 1 (junio de 2019): 37-62. ISSN 2313-9129

ganismos y funcionarios, dentro del marco europeo, ha provocado el traslado de sedes y funcionarios adscritos. La Agencia Europea del Medicamento se ha instalado en Ámsterdam en 2019 con 900 empleados comunitarios, después de un concurso público en el que compitieron Barcelona, Milán, Bratislava y Copenhague, entre otras. Las gestiones diplomáticas tenían que convencer de las bondades de la ciudad con algunos atributos reales y otros intangibles: tolerancia, clima, patrimonio y edificio que albergaría la sede, conexiones internacionales, infraestructura. A su vez, la Autoridad Bancaria Europea tiene previsto trasladarse a París, que gana peso en las finanzas europeas y en la regulación y supervisión del sector bancario europeo. En el ámbito privado, multitud de empresas han movido o duplicado su sede de Londres para mantenerse en territorio de la Unión Europea. En prensa, Lloyds ha abierto una sede en Bruselas; Goldman Sachs ha anunciado a sus empleados que algunos serán trasladados; UBS se ha decantado por Madrid y Frankfurt; el británico HSBC se moverá a París, y la lista continua. Por su parte, la región de Madrid y la Cámara de Comercio crearon un comisionado para la captación de empresas e inversiones perjudicadas por el Brexit con una inversión inicial de 1,75 millones de euros. La estrategia Think Madrid explica las ventajas de reubicarse en esa ciudad e informa a los inversores extranjeros sobre las particularidades de política económica.

\section{Eje cultural, idiomático e identitario}

El segundo objetivo de la diplomacia urbana es la proyección de la identidad a través de la lengua, la cultura y las manifestaciones artísticas. Se dirige a la población extranjera, pero tiene efectos en la sociedad local. La dimensión de la cultura conecta a través de las emociones y las experiencias. Los medios de comunicación constituyen el instrumento prioritario para vehicular los mensajes internacionales, así como el cine y la música. La diplomacia cultural de la ciudad se expresa a través de la promoción de mercados y ferias (Madrid ha protagonizado la Feria del Libro de Guadalajara), la organización de festivales (la franquicia de Hay Festival en distintas ciudades del planeta), la financiación (la Oficina de Turismo de Barcelona emplea el film Vicky Cristina Barcelona, de Woody Allen, como un elemento más de la promoción), los circuitos de turismo literario (de las rutas de El Código da Vinci en París a los recorridos por Dublín para conocer la extensa nómina de escritores locales) o bien los proyectos educativos para la inmersión lingüística (en el top 10 europeo, Granada, Valencia, Sevilla, Madrid y Barcelona aparecen como destinos preferentes de los alumnos Erasmus).

La cultura muestra elementos identificados con la realidad y el imaginario de la ciudad. Es la innovación (London First!), la moda (París, capital de la creación), la diversidad (el barrio madrileño de Lavapiés o el SoHo en Nueva York), la arquitectura (la modernidad de Barcelona o la sobriedad de Notre Dame en París). Es posible men- 
Juan Luis Manfredi Sánchez

Tipología de la diplomacia pública de ciudades

cionar el ejemplo de los circuitos de Viena con la casa de Mozart y sus centenarias salas de conciertos, el recorrido punk de Londres con visita obligada a los estudios de Abbey Road o la industria local creada en torno a The Beatles en Liverpool.

La proyección internacional puede estructurarse en torno a efemérides históricas (800 años de la fundación de la Universidad de Salamanca), la conmemoración de un acontecimiento (el Tratado de Roma) o de otro suceso de especial pertinencia (60 años de actividad aeroespacial en Cabo Cañaveral). También se ejecuta en forma de red, ya que "la Diplomacia cultural entre ciudades facilita la acumulación de capital social no solo entre las ciudades, sino también incrementando el conocimiento de las sociedades" (Global Parliament of Mayors, 2016, p. 4). El mismo Camino de Santiago o la etiqueta "Gran Ruta Cultural" promovida por el Consejo de Europa apalancan la diplomacia cultural a través de distintas geografías.

En el plano profesional, la diplomacia pública se emplea para la captación de talento artístico con el ánimo de que se instale en la ciudad. Se pueden mencionar como referencias la actividad de la Casa de Velázquez, que financia la estancia de 40 artistas al año en París, o la Academia de España en Roma, una institución destinada a creadores españoles y latinoamericanos que quieran desarrollar un proyecto en la capital italiana.

La ciudad combina la vertiente cultural con el desarrollo mediante la protección, promoción y expansión de las industrias locales. El arte, el patrimonio, la diversidad son elementos propios de la estrategia diplomática. En esta línea, aparece la promoción del turismo cultural, que muestra la identidad local a visitantes que se llevan una experiencia directa, no mediada, de los activos culturales y patrimoniales. El Camino de Santiago es uno de los pilares de la proyección internacional de España y, sobre todo, de Galicia, región donde se ubica la ciudad de Santiago de Compostela. Se alinea con la imagen exterior tradicional, aquella que se sostiene sobre el arquetipo romántico de patrimonio, cultura y gastronomía. Se trata de una actividad que se encuadra en los mercados globales de turismo cultural de calidad en áreas rurales (Bambi y Barbari, 2014). Fue declarado Patrimonio de la Humanidad por la Unesco (1993) y primera Gran Ruta Cultural por el Consejo de Europa (1987). El Camino se organiza en etapas que recorren la geografía española y que se pueden completarse a pie, a caballo o en bicicleta. A lo largo del recorrido, se encuentran monumentos históricos, espacios religiosos y ciudades monumentales (León, Burgos, Astorga). Cada año, alrededor de 250000 personas (el 45\%, extranjeros) completan las etapas; y son julio, agosto y septiembre los meses con mayor afluencia. Asimismo, el calendario establece una festividad especial, denominada Año Santo Compostelano, cuando el 25 de julio, festividad de Santiago Apóstol, cae en domingo, lo que sucede con una cadencia de 6, 5, 6 y 11 años (el último año jubilar ha sido 2010 y el siguiente es 2021). En estas oportunidades 
se incrementa el número de visitantes. Tal calendario se denomina Xacobeo y es un descriptor recurrente en la prensa y en la comunicación institucional para referirse a la vertiente religiosa, al arte y la herencia cultural, bien sea al patrimonio arquitectónico, bien a la dimensión de la logística del viaje en sí o a los propios peregrinos. La presencia del Camino en la prensa internacional es recurrente cada verano, en temporada alta de visitantes. La llegada de los peregrinos vehicula los mensajes oficiales de cada ciudad o territorio participante en la experiencia del Xacobeo. Macnamara caracteriza este entorno como "nuevos formatos y sitios híbridos de relaciones públicas, publicidad, emplazamiento de producto en una convergencia entre periodismo y relaciones públicas" (2014, p. 747, traducción propia). El propio diseño del Camino ofrece múltiples posibilidades narrativas, no solo periodísticas, sino experienciales.

A su vez, los proyectos políticos de naturaleza nacionalista o populista emplean la diplomacia cultural de la ciudad para proyectar en el exterior sus mensajes y, sobre todo, para reforzar la propuesta en la mente de la audiencia local. Un ejemplo de esto es el consorcio Diplocat, promovido en 2012 por el gobierno catalán para difundir su visión de la realidad política regional.

\section{Eje político: poder e influencia}

En último término, la diplomacia pública procura incrementar el grado de influencia sobre las decisiones que le afectan y que se toman en la esfera política internacional. La idea de poder subyacente es sencilla: puede ser definida como la capacidad modificar las conductas por la vía de la negociación o la imposición de voluntades. La influencia de la diplomacia urbana no puede medirse por el poder normativo convencional (tratados, acuerdos), puesto que la ciudad carece de estas competencias. Para Barber, el poder viene definido por su condición: “Dejemos que las ciudades, las más reticulares e interconectadas de nuestras asociaciones políticas, definidas sobre todo por la colaboración y el pragmatismo, así como por la creatividad y la multiculturalidad, hagan lo que los estados no alcanzan" (2013, p. 4, traducción propia).

La ciudad ejerce la diplomacia pública con efectos políticos en la medida en que institucionaliza su participación en la esfera internacional (La Porte, 2012) y tiene intención política conectada con el interés público (Gregory, 2016). Por su naturaleza, la diplomacia urbana se organiza en redes, que generan un efecto multiplicador de las capacidades de influencia e incrementan la visibilidad de las demandas políticas. El poder en red, según Castells, es la "nueva morfología social de nuestras sociedades", porque "como tendencia histórica, las funciones y los procesos dominantes en la era de la información cada vez se organizan más en torno a redes" (1997, p. 505). La organización reticular proyecta valor comunicativo: "más de cien ciudades se unen contra", "cuarenta alcaldes piden" y otras frases parecidas contribuyen a la difusión de los men- 
Juan Luis Manfredi Sánchez

Tipología de la diplomacia pública de ciudades

sajes. La institución más conocida es Global Parliament of Mayors, que reúne alcaldes para debatir sobre las cuestiones relevantes de la agenda urbana. Sus integrantes se autodefinen como vertebradores de la participación ciudadana a escala global:

\begin{abstract}
El conocimiento de las ciudades es esencial para proveer a los ciudadanos de la oportunidad de participar en los procesos de toma de decisiones locales y para darle[s] la sensación de pertenencia. [...] Los gobiernos locales desempeñan un rol importante en poner a todos los actores locales juntos a trabajar con este propósito: la sociedad civil, las organizaciones y asociaciones ciudadanas, los actores del sector privado -como grupos de interés- son centrales en la conceptualización del gobierno local y juegan un rol catalizador en el desarrollo local (Global Parliament of Mayors, 2016, p. 2).
\end{abstract}

En relación con los temas que interesan a la agenda de diplomacia urbana, encontramos varias constantes. Algunas de ellas son la prevención de los conflictos (migraciones, salud pública), el principio de subsidiariedad y la promoción del poder local, la lucha contra el cambio climático y la protección del medio ambiente.

En primer lugar, en materia de seguridad, ha crecido el papel de la ciudad como promotora de acuerdos, consensos y propuestas de paz en ámbitos muy concretos. Según Overbeek (2007), las grandes urbes pueden contribuir a los procesos de paz y liderar la cooperación en cinco ejes: la capacidad de tender puentes entre partes enfrentadas al ofrecer un terreno neutral, la mediación, la persuasión, el rol de facilitadora, y el entrenamiento y la capacitación. Este planteamiento aparece en la reflexión elaborada por el Global Parliament of Mayors: "Los conflictos tienen repercusión en la esfera local. En consecuencia, las ciudades tienen tradicionalmente relación con varios estadios del conflicto: prevención, resolución y diplomacia postconflicto. Generalmente, la buena gobernanza local empuja hacia el desarrollo económico, lo que se traduce en mayor seguridad" (2016, p. 3, traducción propia).

En el proceso de paz de Oriente Medio, las redes locales de Israel y Palestina han promovido acuerdos concretos dentro de la Municipal Alliance for Peace. El diálogo municipal, instituido en 2005, se presenta como más accesible para el cierre de acuerdos parciales que contribuyan a la construcción de la paz y la convivencia entre los dos países. Según los datos facilitados por la propia alianza en su sitio, ha gestionado alrededor de 90 millones de dólares para las acciones entre ciudadanos y ha promovido la cooperación entre más de 100 organizaciones.

La iniciativa Mayors for Peace constituye una red de 7614 ciudades y tiene como misión la erradicación de las armas nucleares. Impulsada por los municipios de Hiroshima y Nagasaki en 1982, se dedica a asuntos de seguridad y paz, e incluye en su agenda de trabajo el desarrollo sostenible, la crisis de los refugiados y la lucha contra el terrorismo.

Otra dimensión de la seguridad se compone de las emergencias, los desastres naturales, los ataques terroristas y los estragos sociales de la crisis económica. Bajo el 
Austral Comunicación

Volumen 8, número 1 (junio de 2019): 37-62. ISSN 2313-9129

paraguas de la Rockefeller Foundation, aparece la asociación 100 Resilient Cities, que apoya a las ciudades en el diseño de una estrategia resiliente. Según Rodin, "resiliencia es la capacidad que tiene cualquier entidad -un individuo, una comunidad, una organización o un sistema natural- de prepararse para la disrupción, recuperarse del shock y el estrés, y adaptarse y crecer desde esa experiencia disruptiva" (2014, p. 3, traducción propia). Se concreta en medidas tales como la capacidad de flexibilizar los procesos en caso de emergencia, la formación de los servidores públicos, la promoción de la innovación colectiva y la preparación para gestionar las secuelas del cambio climático.

Otra línea de actuación consiste en la gestión de la influencia, esto es, la capacidad de crear y establecer nuevas oportunidades, así como de modelar el discurso en la arena pública. El organismo más conocido que posee esta finalidad es United Cities and Local Government (UCLG). Se presenta en su página web como "portavoz de todos los tipos de gobiernos locales y regionales -cualquiera sea el tamaño de las comunidades a las que sirven-, representando y defendiendo sus intereses en el plano global" (UCLG, 2016). Su misión es clara: "Ser la voz unida y representación mundial de los gobiernos locales autónomos y democráticos, promoviendo sus valores, objetivos e intereses, a través de la cooperación entre los gobiernos locales y regionales, y ante la vasta comunidad internacional" (UCLG, 2016).

La influencia en red también se da por países o bien organismos supranacionales. Dentro de la propia Unión Europea, las oficinas de representación local o regional son habituales. Se suele atribuir a la ciudad de Birmingham la primera oficina formal ante la entonces Comunidad Económica Europea, aunque rápidamente las comunidades autónomas españolas, los Länder alemanes y las regiones italianas se instalaron en Bruselas y Estrasburgo (Ammassari, 2010), bien por medios propios o bien mediante agencias de desarrollo "para saltarse las limitaciones legales a la representación exterior que imponían los gobiernos nacionales" (Trobbiani, 2016, p. 22).

En el Reino Unido, Core Cities (2018) es otro ejemplo de acción política urbana. Las ciudades de Birmingham, Bristol, Cardiff, Glasgow, Leeds, Liverpool, Manchester, Newcastle, Nottingham y Sheffield representan el 26,5\% de la economía británica y el 29,8\% de la población. En su plan de acción persiguen un modelo económico más transparente, productivo e inclusivo, de modo que reporte beneficios para la ciudadanía, y no solo los capitales. Las ciudades quieren influir en la esfera internacional, porque las decisiones políticas no son exclusivas del gobierno central.

Una de las campañas más interesantes es la reciente Declaración municipalista de los gobiernos locales por el derecho a la vivienda y el derecho a la ciudad en julio de 2018. Se trata de una iniciativa en sintonía con los Objetivos de Desarrollo Sostenible en lo que se refiere a lograr poblaciones seguras, inclusivas, sostenibles y resilientes. En la práctica, busca frenar la desigualdad en el acceso a la vivienda digna, levantar barreras 
Juan Luis Manfredi Sánchez

Tipología de la diplomacia pública de ciudades

al turismo desaforado y la gentrificación de los centros, así como la convivencia pacífica. Esta iniciativa persigue influir en el desarrollo de las políticas urbanísticas, el diseño de infraestructuras y la llegada de migraciones.

En tercer lugar, con respecto al cambio climático y el medio ambiente, existe una multitud de propuestas. La red de ciudades $\mathrm{C} 40$ protagoniza la lucha contra el primero. Esta organización se creó en 2005, a partir de la propuesta del entonces alcalde de Londres, Ken Livingstone, quien convocó a las mayores ciudades del G20 para afrontar este asunto, que no estaba en la agenda de los primeros ministros en las reuniones de aquellos años. En ese período, la institución ha organizado 8000 acciones de distinta naturaleza por el valor de 2800 millones de dólares. La red representa un contrapoder del Estado nación en el diseño y la aplicación de las políticas públicas referidas al cambio climático. Cuenta con 91 ciudades que conforman el 25\% del PBI mundial y congregan a 650 millones de personas. Existen numerosas medidas concretas que sirven para promover el comportamiento sostenible y, entre otras cosas, la aminoración del uso del carbono: autobuses eléctricos, reducción del tráfico en el centro urbano, mejor eficiencia energética de los edificios de titularidad municipal, promoción de la bicicleta, mejora de la accesibilidad peatonal, gestión de los residuos o del agua. Todas estas medidas, no exhaustivas, requieren de la comunicación pública y la relación con la ciudadanía.

En el seno de la Unión Europea, es posible destacar varias propuestas. Por un lado, la Environmental Conference of the European Regions (ENCORE) reúne a representantes y cargos electos de la esfera regional para el desarrollo de políticas medioambientales. Por otro, el International Council for Local Environmental Initiatives (ICLEI) se dedica a la transformación sostenible de los entornos urbanos mediante iniciativas de regulación multinivel. 1500 ciudades y regiones se han alistado en esta propuesta de gestión pública responsable ante el cambio climático, el proceso de migración a las ciudades y la creación de nuevas desigualdades en la comunidad urbana. A su vez, la iniciativa Istanbul Water Consensus (2009) plantea la necesidad de agua salubre en los núcleos urbanos y regionales. Se plantea que el agua es una política pública de naturaleza subestatal. En la misma línea de actuación se encuentra la Network of Regional Governments for Sustainable Development (nrg4SD), que es una red para proteger la biodiversidad, apoyar el desarrollo sostenible y luchar contra el cambio climático. Creada en 2002, cuenta con 50 miembros para defender sus proyectos ante Naciones Unidas y la Unión Europea.

Por último, se relaciona con el cambio climático el auge por la protección de la salud pública global. Dado que las migraciones son imparables, es pertinente cuidar la salud en la ciudad y los asentamientos. En este sentido, destaca la iniciativa WHO European Healthy Cities Network, fundada en 1988, en el marco de Naciones Unidas. 
Austral Comunicación

Volumen 8, número 1 (junio de 2019): 37-62. ISSN 2313-9129

\section{Conclusiones}

El trabajo de investigación se presenta como una primera aproximación al uso de la diplomacia pública por parte de las ciudades como una estrategia de comunicación política internacional. Con respecto a la primera hipótesis, en la que se planteaba la creatividad de las ciudades en el ejercicio de la diplomacia pública por encima de la acción tradicional del Estado, se han expuesto numerosos ejemplos (ver anexo 1) de actividad individual y en red de aquellas, por lo que se demuestra su capacidad efectiva para gestionar, influir y transformar la política exterior. En ninguno de los casos mencionados la ciudad cuenta con competencia real, según el derecho internacional clásico.

Para la segunda hipótesis, se han encontrado tres variables preferentes para la gestión de la diplomacia pública de las ciudades, ya que es una agenda más diversa y flexible: no todas las decisiones están reguladas por tratados o acuerdos internacionales. La economía, la cultura y la política estructuran la actividad, que cada vez es más extensa. La estrategia de comunicación y la gestión de la reputación y los intangibles soportan la demanda ciudadana de una globalización más cercana, más subsidiaria de la política pública municipal.

En último lugar, la tercera hipótesis planteaba la efectividad de la incidencia urbana en los asuntos diplomáticos. Esto se valida, en tanto que las ciudades persiguen no solo la actividad comunicativa, sino también la gestión y la transformación real de su entorno. El cambio climático y las tecnologías han acelerado el interés por la recuperación de competencias en asuntos que afectan a la vida cotidiana del ciudadano.

Para concluir, este trabajo aspira a abrir nuevas vías de investigación que permitan examinar el impacto de las ciudades en la comunicación política internacional, la diplomacia pública y la gestión de la marca país. La ciudad es un sujeto, una unidad de estudio excelente para los nuevos desafíos.

\section{Referencias}

Acuto, M. (2016). Give cities a seat at the top of the table. Nature, 537, 611-613.

Acuto, M., Morissette, M.y Tsouros, A. (2017). City Diplomacy: Towards More Strategic Networking? Learning with WHO Healthy Cities. Global Policy, 8, 14-22.

Ammassari, G. P. (2010). The role of local government bodies in European policymaking. International Review of Sociology, 20(3), 445-456.

Arias Maldonado, M. (2016). La digitalización de la conversación pública: redes sociales, afectividad política y democracia. Revista de Estudios Políticos, 173, 27-54. Obtenido de http://dx.doi.org/10.18042/cepc/rep.173.01.

Asamblea General de Socios (2016). Alianzas para la Nueva Agenda Urbana (GAP, 2016). Documento de posición de la Asamblea General de Socios de Hábitat III. 
Obtenido de http://www.worldurbancampaign.org/sites/default/files/gap_giz dina5_esp_rz_ansicht_0.pdf.

Bambi, G. y Barbari, M. (eds.) (2014). The European Pilgrimage Routes for promoting sustainable and quality tourism in rural areas. Florencia: Firenze University Press.

Barber, B. (2013). If Mayors ruled the world. Nueva Haven: Yale University Press.

Bauman, Z. y Bordoni, C. (2016). Estado de crisis. Barcelona: Paidós.

Borafull, I. (2018). Un nuevo sistema operativo para las ciudades basado en el talento. Vanguardia Dossier: El poder de las ciudades, 67, 42-47.

Bull, H. (1995). The Anarchical Society. Nueva York: Columbia University Press.

Caldwell, N. y Freire, J. R. (2004). The Differences between Branding a Country, a Region and a City: Applying the Brand Box Model. Brand Management, 12, 50-61.

Carroll, W. K. y Hackett, R. A. (2006). Democratic media activism through the lens of social movement theory. Media, Culture \& Society, 28(1), 83-104.

Castells, M. (1997). La era de la información. La sociedad red. Madrid: Alianza Editorial.

Castells, M. (2009). Communication Power. Oxford: Oxford University Press.

Choi, S. y Cai, L. A. (2016). Dimensionality and associations of country and destination images and visitor intention. Place Branding and Public Diplomacy, 12(4), 268-284.

Cooper, A. F., Heine, J. y Thakur, R. (2013). The Oxford Handbook of Modern Diplomacy. Oxford: Oxford University Press.

Copeland, D. (2009). Guerrilla diplomacy: rethinking international relations. Boulder: Lynne Rienner Publishers.

Core Cities (2018). Core Cities UK 2030. Global Success, Local Prosperity. Manchester: Core Cities. Obtenido de https://www.corecities.com/cities/agenda/devolution/ core-cities-uk-2030-global-success-local-prosperity

Cornago, N. (2013). Plural Diplomacies: Normative Predicaments and Functional Imperatives. Ámsterdam: Martinus Nijhoff Publishers.

Cull, N. J. (2008). Public Diplomacy: Taxonomies and Histories. The ANNALS of the American Academy of Political and Social Science, 616(1), 31-54.

Curtis, S. (2014). The power of cities in international relations. Londres: Routledge.

Del Fresno, M., Daly, A. J. y Segado, S. (2016). Identifying the new Influences in the Internet Era: Social Media and Social Network Analysis. Revista Española de Investigaciones Sociológicas, 153, 23-42.

Florida, R. (2003). The Rise of the Creative Class. Nueva York: Basic Books.

Global Parliament of Mayors (2016). Cities as Governance Partners in an Interdependent World. La Haya: Global Parliament of Mayors. Obtenido de https:// globalparliamentofmayors.org/wp-content/uploads/2017/01/Position-PaperGovernance.pdf.

Gonesh, A. y Melissen, J. (2005). Public Diplomacy: Improving Practice. La Haya: 
Austral Cmunicación

Volumen 8, número 1 (junio de 2019): 37-62. ISSN 2313-9129

Netherlands Institute of International Relations Clingendael.

Gregory, B. (2016). Mapping Boundaries in Diplomacy's Public Dimension. The Hague Journal of Diplomacy, 11(1), 1-25.

Harvey, D. (2013). Ciudades rebeldes. Madrid: Akal.

Hocking, B. (2006). Multistakeholder Diplomacy: Forms, Functions, and Frustrations. En Kurbalija, J. y Katrandjiev, V. (eds.). Multistakeholder Diplomacy: Challenges and Opportunities (pp. 13-29). Malta/Ginebra: DiploFoundation.

International Congress and Convention Association (2018). ICCA Statistics Report 2017. Ámsterdam: ICCA. Obtenido de https://www.iccaworld.org/dcps/doc. cfm?docid $=2241$.

La Porte, T. (2012). The Impact of 'Intermestic' Non-State Actors on the Conceptual Framework of Public Diplomacy. The Hague Journal of Diplomacy, 7(4), 441-458.

Lemmert, J. B. (1981). Does mass communication change public opinion after all? A new approach to effect analysis. Chicago: Nelson-Hall.

Lien, D. y Lo, M. (2017). Economic impacts of cultural institutes. The Quarterly Review of Economics and Finance, 64, 12-21.

Macnamara, J. (2014). Journalism-PR relations revisited: the good news, the bad news, and insights into tomorrow's news. Public Relations Review, 40, 739-750.

Manfredi Sánchez, J. L. (2011). Hacia una teoría comunicativa de la diplomacia pública. Communication and Society, 24(2), 150-166.

Manfredi Sánchez, J. L. (2014). Taxonomía de la diplomacia digital en la agenda de las nuevas relaciones internacionales. Historia y Comunicación Social, 19, 341-354.

Manfredi Sánchez, J. L. (2018). Diplomacia corporativa: la nueva inteligencia directiva. Barcelona: UOC.

Manfredi Sánchez, J. L., Herranz de la Casa, J. M. y Calvo Rubio, L. M. (2017). Transparencia y diplomacia: nuevas demandas sociales y rutinas profesionales. Revista Latina de Comunicación Social, 72, 832-848.

Manin, B. (1998). Los principios del gobierno representativo. Madrid: Alianza Editorial.

Meerts, P. W. (2015). Diplomatic Negotiation: Essence and Evolution. La Haya: Clingendael Institute.

Melucci, A. (1989). Nomads of the Present. Londres: Hutchinson Radius.

Mendoza, F. y Vernis, A. (2008). The changing role of governments and the emergence of the relational state. Corporate Governance. The International Journal of Business in Society, 8(4), 389-396.

Nye, J. (2003). La paradoja del poder norteamericano. Madrid: Taurus.

Ociepka, B. (2018). Public Diplomacy as political communication: Lessons from case studies. European Journal of Communication, 33(3), 290-303.

Overbeek, F. (2007). City Diplomacy. The Roles \& Challenges of the peace building 
equivalent of decentralized cooperation. Utrecht: Universiteit Utrecht.

Ramió, C. (2016). El Estado en el año 2050: entre la decadencia y el esplendor. Revista del CLAD Reforma y Democracia, 66, 5-34.

Robinson, L., Helmus, T. C., Cohen, R. S., Nader, A., Radin, A., Magnuson, M. y Migacheva, K. (2018). Modern Political Warfare: Current Practices and Possible Responses. Santa Mónica: RAND Corporation. Obtenido de https://www.rand.org/ pubs/research_reports/RR1772.html.

Rodin, J. (2014). The Resilience Dividend: Being Strong in a World Where Things Go Wrong. Nueva York: Public Affairs.

Sassen, S. (1991). The global city. New York, London, and Tokyo. Nueva Jersey: Princeton University Press.

Schragger, R. (2016). City power. Urban governance in a global age. Oxford: Oxford University Press.

Seib, P. (2012). Real-time diplomacy: politics and power in the social media era. Nueva York: Palgrave Macmillan.

Setzer, J. (2015). Testing the boundaries of subnational diplomacy: the international climate action of local and regional governments. Transnational Environmental Law, 4(2), 319-337.

Silva, A. (2006). Imaginarios urbanos. Bogotá: Tercer Mundo Editores.

Trobbiani, R. (2016). European regions in Brussels: towards functional interest representation? Bruges Political Research Papers, 53. Brujas: College of Europe. Obtenido de https://www.coleurope.eu/system/files_force/research-paper/wp53_ trobbiani.pdf?download $=1$.

United Cities and Local Government (2016). El compromiso de Bogotá y la agenda de acción. Bogotá: UCLG.

United Nations, Department of Economic and Social Affairs, Population Division (2018). World Urbanization Prospects: The 2018 Revision, Methodology. Documento de trabajo ESA/P/WP.252. Nueva York: Naciones Unidas. Obtenido de https:// population.un.org/wup/Publications/Files/WUP2018-Methodology.pdf.

Van der Pluijm, R. (2007). City Diplomacy. The Expanding Role of Cities in International Politics. La Haya: Netherlands Institute of International Relations Clingendael.

Wang, J. (2006). Localising public diplomacy: The role of sub-national actors in nation branding. Place Branding and Public Diplomacy, 2(1), 32-42.

Zenker, S. y Braun, E. (2010, junio). Branding a city: a conceptual approach for place branding and place brand management. Ponencia presentada en $39^{\mathrm{a}}$ Conferencia Anual de la Academia Europea de Marketing, Copenhague, Dinamarca.

Zeraoui, Z. y Castillo Villar, F. (2016). La paradiplomacia de la ciudad. Una estrategia de desarrollo urbano. Revista del CLAD Reforma y Democracia, 65, 225-242. 
Austral Comunicación

Volumen 8, número 1 (junio de 2019): 37-62. ISSN 2313-9129

Anexo 1. Tabla resumen de las iniciativas

\begin{tabular}{|c|c|}
\hline Ejes de actividad & Ejemplo o referencia \\
\hline \multicolumn{2}{|l|}{ Eje económico } \\
\hline Turismo monumental & Gestión del Coliseo de Roma \\
\hline Turismo de tradiciones & Semana Santa de Sevilla \\
\hline Turismo de diseño & Cabina telefónica roja de Londres \\
\hline Diseño & Madrid Design Festival \\
\hline Gastronomía & Queso Stilton \\
\hline Patrimonio intangible & Plaza central de Marraquech \\
\hline Museos & Museo Guggenheim de Bilbao \\
\hline Patrimonio monumental & Palmira, Hatra, Mérida o Edimburgo \\
\hline Cine & Festivales: San Sebastián, Sundance, Cannes; Film Commissions \\
\hline Música & Concierto de Woodstock, Festival Sónar de Barcelona \\
\hline Turismo experiencial & Camino de Santiago \\
\hline Congresos & Barcelona Mobile Week Congress \\
\hline MBA City Monitor & $\begin{array}{l}\text { Boston, Nueva York, Londres, París, Chicago, Toronto, Barcelona, } \\
\text { Carolina del Norte, Singapur, Filadelfia }\end{array}$ \\
\hline Hubs de emprendimiento & Toronto, Montreal, San Pablo, Londres, Estocolmo, Berlín, Singapur \\
\hline Agencias europeas & Ámsterdam y París \\
\hline \multicolumn{2}{|l|}{ Eje cultural, idiomático e identitario } \\
\hline Ferias & Feria del Libro de Guadalajara \\
\hline Festivales & Hay Festival \\
\hline Rutas best sellers & $\begin{array}{l}\text { Barcelona (Vicky Cristina Barcelona, de Woody Allen), París (Código da } \\
\text { Vinci), Dublín o Sevilla (Juego de Tronos) }\end{array}$ \\
\hline Literatura & Dublín (literatura irlandesa) \\
\hline Idioma e inmersión lingüística & Granada, Valencia, Sevilla, Madrid, Barcelona \\
\hline Innovación digital & London First! \\
\hline Moda & París, capital de la creación \\
\hline Arquitectura & Barcelona moderna, París gótico \\
\hline Música & La Viena de Mozart, el Liverpool de The Beatles, el punk de Londres \\
\hline Universidades & 800 años de la Universidad de Salamanca \\
\hline Conmemoración de acontecimientos & Tratado de Roma, lanzamientos de Cabo Cañaveral \\
\hline Academias & Casa de Velázquez, Academia de España en Roma \\
\hline Gran Ruta Cultural & Camino de Santiago \\
\hline
\end{tabular}


Juan Luis Manfredi Sánchez

Tipología de la diplomacia pública de ciudades

\begin{tabular}{ll}
\hline Eje político & \\
\hline Diplomacia subestatal & Diplocat: el consorcio catalán \\
\hline Parlamento & Global Parliament of Mayors \\
\hline Oriente Medio & Alliance for Peace in the Middle East \\
\hline Paz nuclear & Mayors for Peace \\
\hline Seguridad y desastres & 100 Resilient Cities \\
\hline Ciudades globales & United Cities and Local Government \\
\hline Lobby en instituciones europeas & Birmingham \\
\hline Lobby económico & Core Cities UK \\
\hline Cambio climático & Alianza C40 \\
\hline Política medioambiental & Environmental Conference of the European Regions \\
\hline Transformación sostenible & International Council for Environmental Initiatives \\
\hline Gestión del agua & Istanbul Water Consensus \\
\hline Desarrollo sostenible & Network of Regional Governments for Sustainable Development \\
\hline Salud público global & WHo European Healthy Cities Network \\
\hline
\end{tabular}

